Research Article

\title{
Systematic Elucidation of the Potential Mechanisms of Core Chinese Materia Medicas in Treating Liver Cancer Based on Network Pharmacology
}

\author{
Zhulin Wu $\mathbb{D}$, ${ }^{1,2}$ Lina Yang, ${ }^{2}$ Li He, ${ }^{2}$ Lianan Wang, ${ }^{2}$ and Lisheng Peng $\mathbb{D}^{2}$ \\ ${ }^{1}$ The Fourth Clinical Medical College of Guangzhou University of Chinese Medicine, Shenzhen 518033, China \\ ${ }^{2}$ Shenzhen Traditional Chinese Medicine Hospital, Shenzhen 518033, China \\ Correspondence should be addressed to Lisheng Peng; szlisheng_peng@163.com
}

Received 14 November 2019; Revised 23 March 2020; Accepted 26 March 2020; Published 24 April 2020

Academic Editor: Carmen Mannucci

Copyright (c) 2020 Zhulin Wu et al. This is an open access article distributed under the Creative Commons Attribution License, which permits unrestricted use, distribution, and reproduction in any medium, provided the original work is properly cited.

Objective. In this study, the data mining method was used to screen the core Chinese materia medicas (CCMMs) against primary liver cancer (PLC), and the potential mechanisms of CCMMs in treating PLC were analyzed based on network pharmacology. Methods. Traditional Chinese medicine (TCM) prescriptions for treating PLC were obtained from a famous TCM doctor in Shenzhen, China. According to the data mining technique, the TCM Inheritance Support System (TCMISS) was applied to excavate the CCMMs in the prescriptions. Then, bioactive ingredients and corresponding targets of CCMMs were collected using three different TCM online databases, and target genes of PLC were obtained from GeneCards and OMIM. Afterwards, common targets of CCMMs and PLC were screened. Furthermore, a network of CCMMs bioactive ingredients and common target gene was constructed by Cytoscape 3.7.1, and gene ontology (GO) and signaling pathways analyses were performed to explain the mechanism of CCMMs in treating PLC. Besides, protein-protein interaction (PPI) analysis was used to identify key target genes of CCMMs, and the prognostic value of key target genes was verified using survival analysis. Results. A total of 15 high-frequency Chinese materia medica combinations were found, and CCMMs (including Paeoniae Radix Alba, Radix Bupleuri, Macrocephalae Rhizoma, Coicis Semen, Poria, and Curcumae Radix) were identified by TCMISS. A total of 40 bioactive ingredients (e.g., quercetin, kaempferol, and naringenin) of CCMMs were obtained, and 202 common target genes of CCMMs and PLC were screened. GO analysis indicated that biological processes of CCMMs were mainly involved in response to drug, response to ethanol, etc. Pathway analysis demonstrated that CCMMs exerted its antitumor effects by acting on multiple signaling pathways, including PI3K-Akt, TNF, and MAPK pathways. Also, some key target genes of CCMMs were determined by PPI analysis, and four genes (MAPK3, VEGFA, EGF, and EGFR) were found to be correlated with survival in PLC patients. Conclusion. Based on data mining and network pharmacology methods, our results showed that the therapeutic effect of CCMMs on PLC may be realized by acting on multitargets and multipathways related to the occurrence and development of PLC.

\section{Introduction}

Primary liver cancer (PLC) is one of the most common and fatal malignant tumors. In 2012, 782,500 new cases of PLC were reported worldwide, being responsible for an estimated 745,000 deaths globally, and PLC ranked as the second leading cause of death from cancer [1, 2]. Moreover, the 5-year survival rate for PLC patients remains low [3]. The most common type of PLC is hepatocellular carcinoma (HCC), which accounts for approximately
$70 \%-85 \%$ of all PLC [4]. The risk factors for PLC contain hepatitis $\mathrm{C}$ virus (HCV) or hepatitis B virus (HBV) infection, excess alcohol consumption, and aflatoxins. As most patients are diagnosed at an advanced stage and lose the opportunity for surgical resection, the majority of them can only receive palliative treatment. For decades, few treatments have been able to effectively improve the prognosis of advanced PLC, and most of the chemotherapeutic drugs used in advanced PLC have toxic or side effects $[5,6]$. 
Due to the limitations of present therapies, complementary and alternative medicines have been more utilized for the treatment of PLC in the past few decades [7]. In China, the treatment of cancer with traditional Chinese medicine (TCM) has a very long history. TCM doctors are trying to use Chinese materia medica as an adjuvant therapy to ameliorate the quality of life or survival time of patients with PLC. A retrospective study indicated that a combination of Chinese materia medicas and conventional therapies may improve the survival of patients with intermediate or advanced PLC [8]. Several TCM prescriptions have also been confirmed to have anti-PLC effects in basic and clinical research [9-11]. Furthermore, a metaanalysis found that TCM combined with chemotherapy showed significant efficacy and safety in objective response rate, survival time extension, improvement for life quality, and reduction of therapeutic toxicity [7]. Besides, a study reported a rare case of recurrent PLC patient with complete regression of the target lesion with 2 years treatment of Chinese materia medicas [12]. Taken together, as a part of TCM, Chinese materia medicas could effectively improve the quality of life and prolong the survival time in patients with PLC. However, the use of Chinese materia medicas in the treatment of PLC was mostly based on doctors' experience, and it is still unclear which combination of Chinese materia medicas may be effective in treating PLC. In addition, due to the complicated components of Chinese materia medicas, the specific mechanism of Chinese materia medicas in the treatment of PLC is not entirely clear.

At present, the network pharmacology approach can be used to interpret the relationship between diseases, drugs, and targets, showing the network of drug targets from a holistic view [13]. Also, it is of great significance to understand the polypharmacology of drugs and effects of drugs on biological networks using the network pharmacology approach [14]. Additionally, the data mining method has been widely used in TCM, and data mining analysis of prescription is also one of the research hotspots in TCM [15]. Moreover, the data mining method can be utilized to study the rules of Chinese materia medicas in treating diseases and identifying core Chinese materia medicas (CCMMs) from a large number of TCM prescriptions. In this study, the data mining method was used to extract the CCMMs for the treatment of PLC, and we tried to uncover potential mechanisms for CCMMs as a potential therapy for PLC using the network pharmacology approach.

\section{Materials and Methods}

First of all, TCM prescriptions for the treatment of PLC were collected from the clinical practice of a famous TCM doctor, and then a database of TCM prescriptions for treating PLC was established. Secondly, data mining software named the TCM Inheritance Support System (TCMISS) was used to extract the CCMMs. Subsequently, the ingredients and target genes of the CCMMs were obtained from three different databases. Target genes related to PLC were searched in GeneCards and OMIM databases. In addition, the common target genes of CCMMs and PLC were screened. Furthermore, potential mechanism of CCMMs in treating liver cancer was analyzed, and survival analyses of key target genes were performed. The overall flowchart for this study is displayed in Figure 1.

2.1. Prescriptions Data Collation. All TCM prescriptions for the treatment of PLC were acquired from Shenzhen traditional Chinese Medicine Hospital affiliated to Guangzhou University of Chinese Medicine (date: from October 2009 to August 2019), which were prescribed by Professor Chensheng Ouyang, a famous TCM doctor in Shenzhen, China. The inclusion criterion of TCM prescriptions was the first diagnosis was PLC, and exclusion criteria were (1) duplicate prescriptions of the same patients; (2) prescriptions were primarily for treating acute symptoms such as cold, cough, diarrhea, and vomiting; and (3) external prescriptions. Based on data mining methods, TCMISS was applied to extract the high-frequency Chinese materia medicas and CCMMs of the prescriptions. TCMISS is software widely used in TCM prescription data analyses, which integrates general statistics, text mining, association rules, and complex system entropy clustering methods $[15,16]$. Through the "prescription management" module in the "platform management system" of TCMISS, the prescriptions data were entered. To ensure the reliability and accuracy of the results, two authors (Yang and $\mathrm{He}$ ) examined the data after completion of the data entry.

2.2. Data Mining of Prescriptions and Extraction of CCMMs. The association rules method in TCMISS was used to identify the high-frequency combinations of Chinese materia medicas, and then CCMMs were obtained. Specifically, the "prescription analysis" module of "data analysis" section in TCMISS was selected to identify the frequency and the combinations of Chinese materia medicas. Analysis of prescription rules was carried out under the condition that support the degree was 200 and the confidence score was greater than or equal to 0.95 .

2.3. Bioactive Ingredients and Targets of CCMMs. TCM Systems Pharmacology Database and Analysis Platform (TCMSP, http://tcmspw.com/tcmsp.php) [17], Integrative Pharmacology-based Research Platform of TCM (TCMIP, http://www.tcmip.cn/) [18], and Bioinformatics Analysis Tool for Molecular Mechanism of TCM (BATMAN-TCM, http://bionet.ncpsb.org/batman-tcm/) [19], were utilized to retrieve the ingredients of CCMMs. Each ingredient was annotated using the Mol ID from TCMSP, and the parameters for screening bioactive ingredients were set as follows: drug-likeness (DL) $\geq 0.18$ and oral bioavailability $(\mathrm{OB}) \geq 30 \%$ [20]. Besides, targets related to bioactive ingredients were obtained by TCMSP. All target names were converted into corresponding gene names using UniProt (https://www.uniprot.org/). 


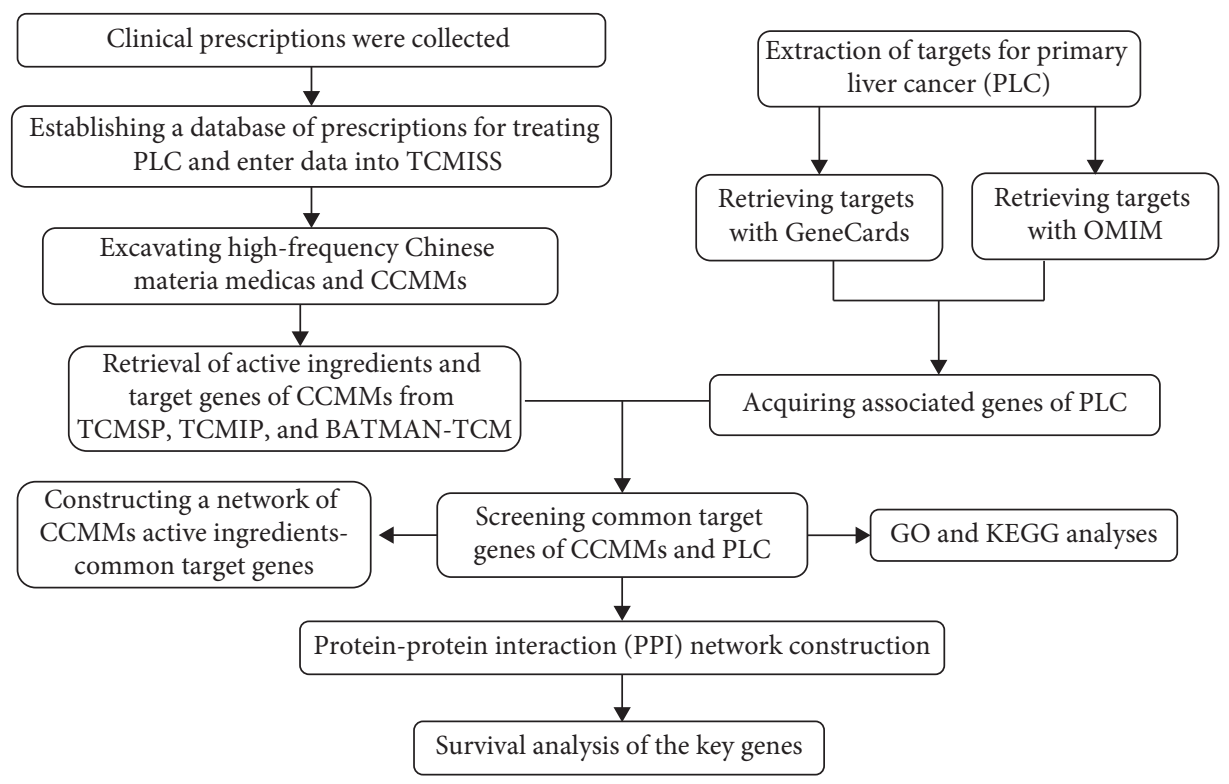

Figure 1: Flow diagram showing the process of the present study.

2.4. Prediction of Target Genes of PLC. The databases "GeneCards" (https://www.genecards.org/) [21] and "Online Mendelian Inheritance in Man" (OMIM, https:// omim.org/) [22] were searched using keywords primary liver cancer, hepatocellular carcinoma; HCC; hepatoma; hepatic cancer; and hepatic carcinoma. Potential therapeutic target genes of PLC were gained after summarizing and eliminating duplication.

2.5. The Network of CCMMs Bioactive Ingredients and Common Target Genes. Common target genes of CCMMs and PLC were screened using the Draw Venn Diagram online tool (http://bioinformatics.psb.ugent.be/webtools/ Venn/). In order to fully understand the molecular mechanism, the network of CCMMs bioactive ingredients and common targets was constructed by using Cytoscape 3.7.1.

2.6. Gene Ontology (GO) and Signaling Pathway Analyses. GO and Kyoto Encyclopedia of Genes and Genomics (KEGG) signaling pathway analyses for common target genes were performed by the Database for Annotation, Visualization, and Integrated Discovery (DAVID, https:// david.ncifcrf.gov/) [23]. In addition, GO enrichment analysis was performed according to three types, including GO biological processes (GO-BP), GO cellular component (GO$\mathrm{CC}$ ), and GO molecular function (GO-MF). The results of GO analysis were presented by a barplot using "ggplot2" package in $R$ software (version 3.6.0). Furthermore, top 20 key KEGG pathways were identified according to the order of gene count. In the light of the biological functions of common targets and correlative signaling pathways, the mechanism of CCMMs against PLC was discussed. In GO and KEGG analyses, a false discovery rate (FDR) below 0.05 was considered significant.
2.7. Protein-Protein Interaction (PPI) Analysis and Screening for Key Genes. The common target genes were input into Search Tool for the Retrieval of Interacting Genes (STRING) database (https://string-db.org/) [24] to construct a proteinprotein interaction (PPI) network by setting the minimum required interaction score at 0.400 . Furthermore, CytoNCA plugin [25] in Cytoscape 3.7.1 was used to perform a topological analysis, including degree centrality (DC), betweenness centrality (BC), and closeness centrality (CC), for key genes screening. The core PPI network was presented by using NetworkAnalyzer plugin in Cytoscape.

2.8. Correlation between Key Genes and Prognosis. After obtaining the key target genes from the PPI network, we used the Kaplan-Meier Plotter (http://kmplot.com/analysis/) to explore the association between key genes expression and prognosis in patients with PLC. The Kaplan-Meier Plotter includes data on survival prognosis of liver cancer in The Cancer Genome Atlas (TCGA) database [26]. In the present study, survival analysis was performed for key genes with DC $>90$, and overall survival (OS) was analyzed by the Kaplan-Meier (K-M) survival analysis (log-rank test). A p value less than 0.05 was considered significant.

\section{Results}

3.1. Results of Data Mining by TCMISS. A total of 326 TCM prescriptions for the treatment of PLC were collected. The most frequently used Chinese materia medicas were Paeoniae Radix Alba, Radix Bupleuri, Macrocephalae Rhizoma, Coicis Semen, Poria, Curcumae Radix, Dioscoreae Rhizoma, and curcumae rhizome. Top 20 frequently-used Chinese materia medicas were shown in Table 1 . In addition, 15 most commonly used combinations of Chinese materia medicas were acquired, including 6 kinds of Chinese materia medicas, and the top 15 combinations of Chinese materia 
TABLE 1: Top 20 Chinese materia medicas in all TCM prescriptions.

\begin{tabular}{|c|c|c|c|c|c|}
\hline Number & $\begin{array}{l}\text { Chinese materia } \\
\text { medicas }\end{array}$ & Freq & Number & $\begin{array}{l}\text { Chinese } \\
\text { materia } \\
\text { medicas }\end{array}$ & Freq \\
\hline 1 & $\begin{array}{c}\text { Paeoniae radix } \\
\text { alba }\end{array}$ & 301 & 11 & $\begin{array}{l}\text { Amomi } \\
\text { fructus }\end{array}$ & 123 \\
\hline 2 & Radix bupleuri & 292 & 12 & $\begin{array}{l}\text { Codonopsis } \\
\text { radix }\end{array}$ & 116 \\
\hline 3 & $\begin{array}{l}\text { Macrocephalae } \\
\text { rhizoma }\end{array}$ & 279 & 13 & Eupolyphaga & 114 \\
\hline 4 & Coicis semen & 278 & 14 & $\begin{array}{l}\text { Scutellariae } \\
\text { radix }\end{array}$ & 104 \\
\hline 5 & Poria & 253 & 15 & $\begin{array}{c}\text { Semen } \\
\text { dolichoris } \\
\text { album } \\
\text { Citrus }\end{array}$ & 95 \\
\hline 6 & Curcumae radix & 224 & 16 & $\begin{array}{l}\text { reticulata } \\
\text { blanco }\end{array}$ & 89 \\
\hline 7 & $\begin{array}{l}\text { Dioscoreae } \\
\text { rhizoma }\end{array}$ & 172 & 17 & $\begin{array}{c}\text { Fructus } \\
\text { gardeniae }\end{array}$ & 76 \\
\hline 8 & $\begin{array}{l}\text { Curcumae } \\
\text { rhizoma }\end{array}$ & 166 & 18 & $\begin{array}{l}\text { Amomi } \\
\text { fructus } \\
\text { rotundus }\end{array}$ & 72 \\
\hline 9 & $\begin{array}{l}\text { Trionycis } \\
\text { carapax }\end{array}$ & 160 & 19 & $\begin{array}{l}\text { Astmgali } \\
\text { radix }\end{array}$ & 70 \\
\hline 10 & Fructus aurantii & 127 & 20 & $\begin{array}{l}\text { Moutan } \\
\text { cortex }\end{array}$ & 69 \\
\hline
\end{tabular}

medicas are displayed in Table 2. Moreover, TCMISS was utilized to demonstrate the application mode of Chinese materia medicas in a networked view, and the Chinese materia medicas shown in Figure 2(a) were CCMMs.

3.2. Results of Bioactive Ingredients and Targets of CCMMs. The data of CCMMs ingredients from three different databases are listed in Table 3. Also, bioactive ingredient count of each core Chinese materia medica is presented in Table 3. Following removal of the duplicates, a total of 40 ingredients were identified, and 206 potential target genes of CCMMs were obtained.

3.3. Potential Target Genes of PLC. Fifteen thousand four hundred and seventy-eight and three hundred and thirtyeight PLC-related target genes were extracted from GeneCards and OMIM databases, respectively. A total of 15719 therapeutic target genes for PLC were obtained after the elimination of duplicates.

\subsection{Construction of CCMMs Bioactive Ingredients-Common} Target Genes Network. A total of 202 common targets of CCMMs and PLC were identified by Draw Venn Diagram online tool (Figure 2(b)). A network of CCMMs bioactive ingredients-common target genes including 242 nodes (202 target genes, 40 bioactive ingredients) and 447 edges (interactions) was established (Figure 3), and the details of bioactive ingredients are shown in Table 4.
3.5. Results of GO and KEGG Signaling Pathway Analyses. The top 5 enrichment results in each category of GO analysis are displayed in Figure $4(\mathrm{FDR}<0.05)$. The GO-BP involved 74 enrichment results, including response to drug and response to ethanol, and 23 enrichment results were related to GO-MF which cover the enzyme binding, protein homodimerization activity, etc. In addition, there were 14 enrichment results in GO-CC, including extracellular space and cytosol. Moreover, the results of the top 20 KEGG signaling pathways are listed in Table 4. According to the results of KEGG, the common target genes of PLC and CCMMs were mainly enriched in cancer-related signaling pathways, such as pathways in cancer, PI3K-Akt pathway, TNF pathway, and MAPK pathway (Table 5).

3.6. Results of PPI Network Analysis. The rough PPI network with 201 nodes and 3169 edges was constructed using STRING. Because nodes with DC only more than twofold median DC of all nodes can be significant targets in the PPI network [27], we used twice the median of the DC value to generate a subnetwork. After that, target genes with $\mathrm{BC} \geq$ median and $\mathrm{CC} \geq$ median were selected to construct the core network and obtain key nodes (genes). The screening process is shown in Figure 5(a), and the key genes in the core network is presented in Figure 5(b). The results of PPI analysis demonstrated that AKT1, IL6, MAPK3, VEGFA, and CASP 3 could be considered as key target genes of CCMMs in the treatment of PLC.

3.7. Effect of Key Genes on OS of PLC Patients. The associations between nine key genes (AKT1, IL6, MAPK3, VEGFA, CASP3, JUN, EGF, EGFR, and MYC) with the DC $>90$ and OS rate were analyzed by the K-M method. Survival curves of the key genes are shown in Figures 6(a)6(i). Our data demonstrated that high expressions of MAPK3, VEGFA, and EGF were significantly related to poor prognosis (Figures 6(c), 6(d), 6(h)), and high expression of EGFR was correlated with better prognosis (Figure 6(h)). All other key genes showed no significant difference, Figures 6(a)-6(i).

\section{Discussion}

The results of data mining demonstrated that CCMMs for PLC comprised six Chinese materia medicas, including Radix Alba, Radix Bupleuri, Macrocephalae Rhizoma, Coicis Semen, Poria, and Curcumae Radix. According to TCM theories, these CCMMs can be applied to disperse stagnated liver Qi, reinforce Qi, and nourish Xue (blood). In TCM, the pathogenesis of PLC is usually thought to be caused by liver Qi stagnation and deficiency of qi and Xue. Thus, data mining results are in line with the theories of TCM. Furthermore, the results of network pharmacology revealed that the bioactive ingredients of CCMMs, including quercetin, kaempferol, naringenin, isorhamnetin, beta-sitosterol, and stigmasterol were related to more target genes, which were the key bioactive ingredients. Quercetin has been proved to induce apoptosis of liver cancer cells (SMMC-7721), and its 
TABle 2: Top 15 commonly used combinations of Chinese materia medicas.

\begin{tabular}{lcc}
\hline Number & Most commonly used combinations of Chinese materia medicas & Freq \\
\hline 1 & Paeoniae radix alba, radix bupleuri & 289 \\
2 & Paeoniae radix alba, coicis semen & 263 \\
3 & Radix bupleuri, coicis semen & 256 \\
4 & Macrocephalae rhizoma, paeoniae radix alba & 255 \\
5 & Paeoniae radix alba, radix bupleuri, coicis semen & 254 \\
6 & Macrocephalae rhizoma, radix bupleuri & 246 \\
7 & Macrocephalae rhizoma, paeoniae radix alba, radix bupleuri & 244 \\
8 & Macrocephalae rhizoma, coicis semen \\
9 & Macrocephalae rhizoma, poria & 243 \\
10 & Paeoniae radix alba, poria \\
11 & Macrocephalae rhizoma, paeoniae radix alba, coicis semen \\
12 & Macrocephalae rhizoma, radix bupleuri, coicis semen & 236 \\
13 & Curcumae radix, paeoniae radix alba & 231 \\
14 & Curcumae radix, radix bupleuri \\
15 & Radix bupleuri, poria \\
\hline
\end{tabular}

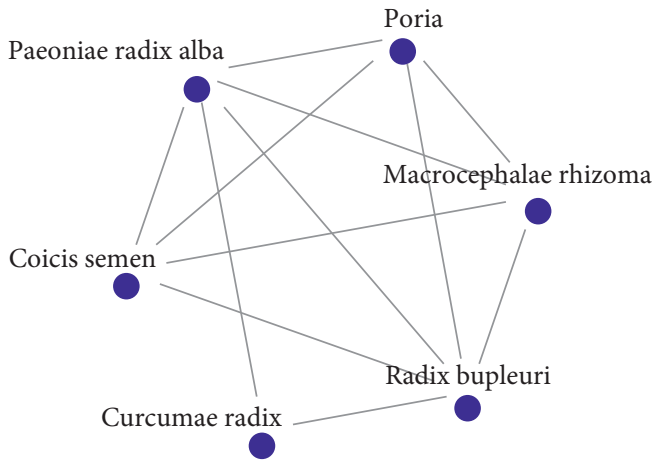

(a)

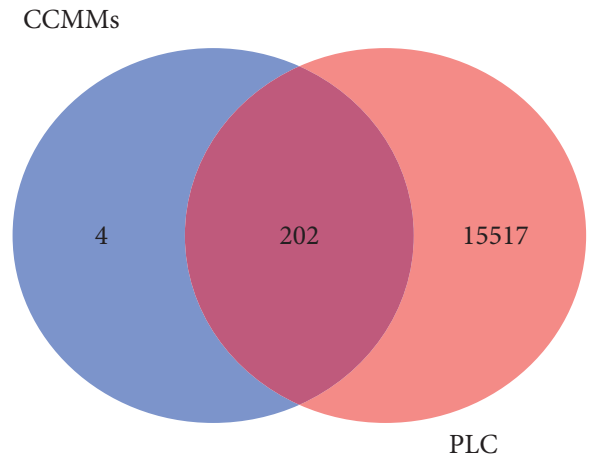

(b)

Figure 2: Network of CCMMs by TCMISS (a) and Venn diagram for common target genes of CCMMs and PLC (b).

TABLE 3: The data of CCMMs ingredients from three different databases.

\begin{tabular}{lcccc}
\hline CCMMs & TCMSP & TCMIP & BATMAN & Bioactive ingredient counts \\
\hline Paeoniae radix alba & 85 & 54 & 35 & 18 \\
Radix bupleuri & 349 & 62 & 82 & 18 \\
Macrocephalae rhizoma & 55 & 20 & 11 & 7 \\
Coicis semen & 38 & 2 & 3 & 9 \\
Poria & 34 & 33 & 21 & 18 \\
Curcumae radix & 222 & 11 & 27 & 15 \\
\hline
\end{tabular}

mechanism could be related to the inhibition of activation of Akt by PTEN gene overexpression [28]. Kaempferol is one of the general flavonoids and has been reported to curb proliferation and migration of liver cancer HepG2 cells through inactivating the PI3K/Akt signaling pathway [29]. Recent research indicated that naringenin could induce apoptosis and regulate the MAPK pathway so as to prevent the occurrence of liver cancer [30]. Choi [31] reported that isorhamnetin could inhibit the proliferation of human hepatocarcinoma Hep3B cells by arresting the cell cycle at the G2/M phase. Zhang [32] found that beta-sitosterol may inhibit proliferation and induced apoptosis of HepG2 cells through membrane death receptor and the mitochondrial pathway. In addition, a study showed that stigmasterol could induce apoptosis of HepG2 cells by upregulating proapoptotic genes (Bax, p53) and downregulating antiapoptotic gene (Bcl-2) expressions [33]. The interaction network of CCMMs bioactive and common targets revealed that CCMMs are able to serve an antitumor role through multiple bioactive ingredients and targets, which is very consistent with the holism and the thought of treatment based on syndrome differentiation in TCM.

In the present study, GO and KEGG analyses were used to predict the underlying mechanism of CCMMs in treating PLC. The result of GO analysis indicated that common targets were significantly enriched in the mitochondrion, extracellular exosome, membrane raft, cytosol, and extracellular space, showing that these genes are involved in 


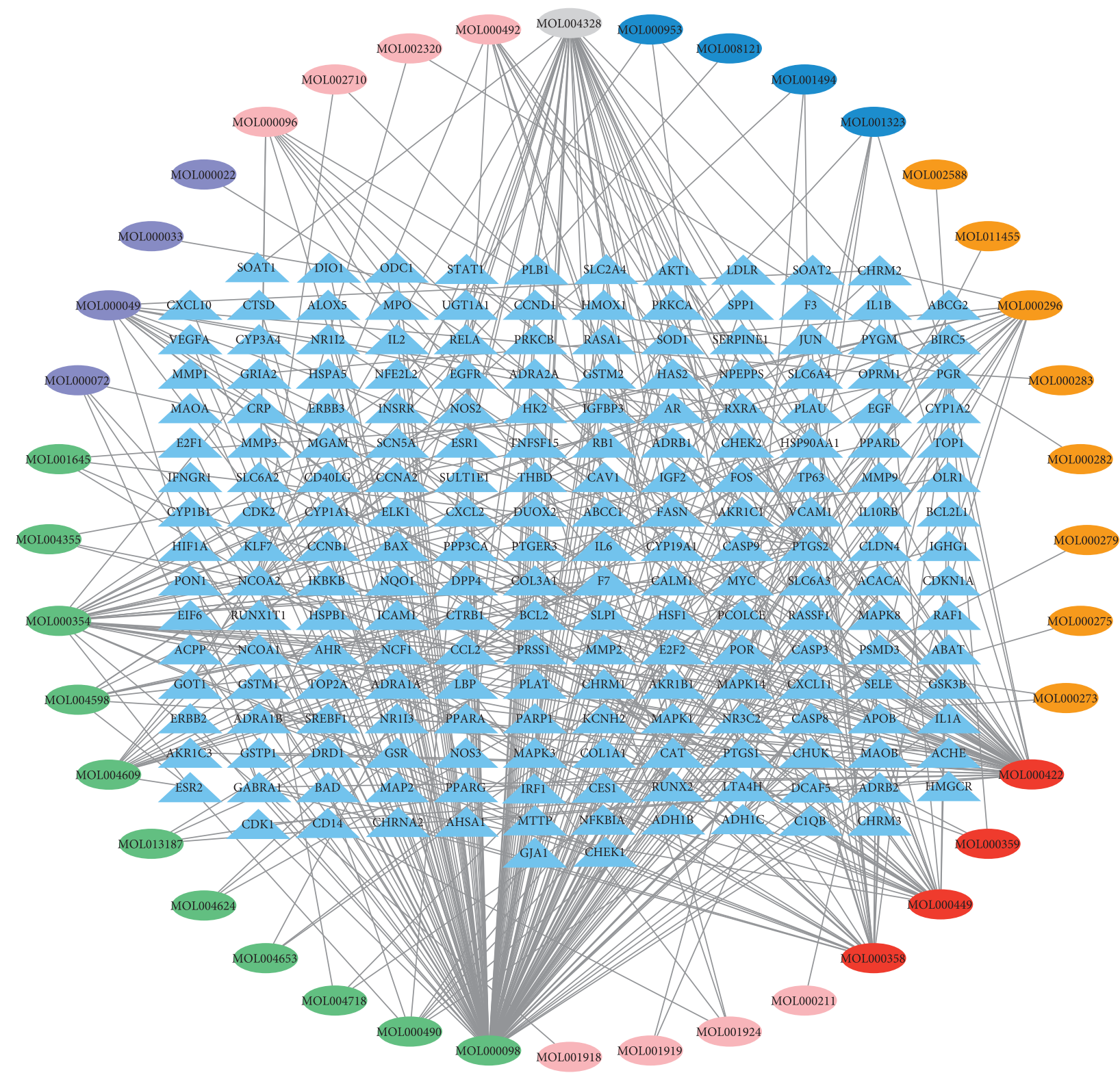

FIGURE 3: The network of CCMMs and common target genes. Light blue triangles represent the common target genes of PLC and CCMMs bioactive ingredients. The ovals indicate the bioactive ingredients of CCMMs, in which red represents the common bioactive ingredients of multiple Chinese materia medicas, and pink, purple, green, orange, blue, and gray represent the bioactive ingredients of Paeoniae Radix Alba, Macrocephalae Rhizoma, Radix Bupleuri, Poria, Coicis Semen, and Curcumae Radix, respectively.

various cell metabolisms. As for GO-MF, the genes were involved in drug binding, identical protein binding, protein binding, protein homodimerization activity, and enzyme binding, which are correlated with the liver metabolic function. In GO-BP, common target genes were most enriched in response to hypoxia, positive regulation of transcription from RNA polymerase II promoter, response to lipopolysaccharide, response to ethanol, and response to drug, suggesting that CCMMs may be associated with cell apoptosis, liver metabolism, immune system, and drug metabolism. KEGG analysis showed that CCMMs may produce anti-PLC effects via multiple pathways, including pathways in cancer, hepatitis B, PI3K-Akt, TNF, MAPK, hepatitis C, HIF-1, toll-like receptor, and Ras signaling pathway. Previous studies have reported that the expression of Akt in PLC cells is significantly higher than that in normal liver tissues [34], and Chinese materia medicas could regulate the PI3K/Akt/mTOR signaling pathway to induce cell apoptosis and inhibit the growth of hepatoma cells [35]. Hence, the PI3K/Akt pathway may provide novel target drugs for HCC treatment. In addition, the literature has shown that there was a positive correlation between HBV infection and PLC, and HBV that integrate in the host genome can lead to the inactivation of tumor suppressor genes or the activation of protooncogenes [36-38]. It has been reported that TNF serves a critical role in the development of liver cancer [39]. Also, HIF-1 plays an important role in immune escape and epithelial-mesenchymal 
TABLE 4: The information of screened bioactive ingredients in CCMMs.

\begin{tabular}{|c|c|c|c|c|}
\hline Mol ID & Bioactive ingredients & $\begin{array}{l}\text { Gene } \\
\text { count }\end{array}$ & OB & $\mathrm{DL}$ \\
\hline MOL000098 & Quercetin & 140 & 46.43 & 0.28 \\
\hline MOL000422 & Kaempferol & 55 & 41.88 & 0.24 \\
\hline MOL004328 & Naringenin & 34 & 59.29 & 0.21 \\
\hline MOL000354 & Isorhamnetin & 30 & 49.6 & 0.31 \\
\hline MOL000358 & Beta-sitosterol & 28 & 36.91 & 0.75 \\
\hline MOL000449 & Stigmasterol & 27 & 43.83 & 0.76 \\
\hline MOL000296 & Hederagenin & 17 & 36.91 & 0.75 \\
\hline MOL000049 & $3 \beta$-Acetoxyatractylone & 14 & 54.07 & 0.22 \\
\hline MOL004609 & Areapillin & 14 & 48.96 & 0.41 \\
\hline MOL004598 & 3,5,6,7-Tetramethoxy-2-(3,4,5-trimethoxyphenyl)chromone & 10 & 31.97 & 0.59 \\
\hline MOL000096 & $(-)$-Catechin & 9 & 49.68 & 0.24 \\
\hline MOL000492 & $(+)$-Catechin & 9 & 54.82 & 0.24 \\
\hline MOL000490 & Petunidin & 8 & 30.05 & 0.31 \\
\hline MOL001323 & Sitosterol alpha1 & 5 & 43.28 & 0.78 \\
\hline MOL000072 & $8 \beta$-Ethoxy atractylenolide III & 4 & 35.05 & 0.21 \\
\hline MOL001645 & Linoleyl acetate & 4 & 42.1 & 0.2 \\
\hline MOL001924 & Paeoniflorin & 4 & 53.87 & 0.79 \\
\hline MOL004653 & (+)-Anomalin & 4 & 46.06 & 0.66 \\
\hline MOL013187 & Cubebin & 4 & 57.13 & 0.64 \\
\hline MOL000359 & Sitosterol & 3 & 36.91 & 0.75 \\
\hline MOL000953 & CLR & 3 & 37.87 & 0.68 \\
\hline MOL001494 & Mandenol & 3 & 42 & 0.19 \\
\hline MOL004355 & Spinasterol & 3 & 46.43 & 0.28 \\
\hline MOL004624 & Longikaurin A & 3 & 47.72 & 0.53 \\
\hline MOL004718 & $\alpha$-Spinasterol & 3 & 42.98 & 0.76 \\
\hline MOL000273 & $\begin{array}{l}\text { (2R)-2-((3S,5R,10S,13R,14R,16R,17R)-3,16-dihydroxy-4,4,10,13,14-pentamethyl- } \\
2,3,5,6,12,15,16,17 \text {-octahydro-1H-cyclopenta(a)phenanthren-17-yl)-6-methylhept-5-enoic acid }\end{array}$ & 2 & 30.93 & 0.81 \\
\hline MOL001919 & Palbinone & 2 & 43.56 & 0.53 \\
\hline MOL002320 & Poriferast-5-en-3beta-ol & 2 & 54.83 & 0.24 \\
\hline MOL002710 & Pyrethrin II & 2 & 54.83 & 0.24 \\
\hline MOL000022 & 14-acetyl-12-senecioyl-2E,8Z,10E-atractylentriol & 1 & 63.37 & 0.3 \\
\hline MOL000033 & $\begin{array}{l}\text { (3S,8S,9S,10R,13R,14S,17R)-10,13-dimethyl-17-((2R,5S)-5-propan-2-yloctan-2-yl)- } \\
\text { 2,3,4,7,8,9,11,12,14,15,16,17-dodecahydro-1H-cyclopenta(a)phenanthren-3-ol }\end{array}$ & 1 & 36.23 & 0.78 \\
\hline MOL000211 & Mairin & 1 & 55.38 & 0.78 \\
\hline MOL000275 & Trametenolic acid & 1 & 38.71 & 0.8 \\
\hline MOL000279 & Cerevisterol & 1 & 37.96 & 0.77 \\
\hline MOL000282 & Ergosta-7,22E-dien-3beta-ol & 1 & 43.51 & 0.72 \\
\hline MOL000283 & Ergosterol peroxide & 1 & 40.36 & 0.81 \\
\hline MOL001918 & Paeoniflorgenone & 1 & 87.59 & 0.37 \\
\hline MOL002588 & Eburicol & 1 & 44.17 & 0.82 \\
\hline MOL008121 & 2-Monoolein & 1 & 34.23 & 0.29 \\
\hline MOL011455 & 20-Hexadecanoylingenol & 1 & 44.17 & 0.83 \\
\hline
\end{tabular}

transformation of PLC [40], which shows great prospects in the treatment of PLC. Furthermore, research indicated that both HBV and HCV-related hepatocarcinogenesis activate the Ras/MAPK pathway which is associated with a poor prognosis [41-43]. Additionally, HCV-induced EGFR-ERK signaling may promote the development and progression of PLC [44]. The toll-like receptor (TLR) pathway is involved in the initiation, progression, and metastasis of liver cancer, which may become a new target for targeted therapies of PLC [45]. Patients with PLC are susceptible to infections due to hypoimmunity, which may be related to tuberculosis, HTLV-I infection, influenza A, and Chagas disease pathways. Besides, other signaling pathways in this study are also associated with cancer. These findings indicated that CCMMs could be used in treating PLC by regulating multiple pathways related to the occurrence and development of cancer.

Several key target genes of CCMMs were identified by PPI network construction, and four of the top nine genes were found to be correlated with OS in PLC patients. An experimental study showed that overexpression of AKT1 may promote the migration and invasion of PLC cells (HepG2) [46]. Inflammatory plays a key role in the occurrence, development, and metastasis of PLC. IL-6 is highly upregulated in PLC, which is correlated with rapid progression from hepatitis to PLC [47]. Previous studies found that CASP3 (Caspase-3) can prevent chemical-induced PLC by suppressing p38 activation and hepatocyte death [48], and total flavonoids from Ampelopsis grossedentata (Teng cha) exerts an antitumor effect on PLC by upregulating the 


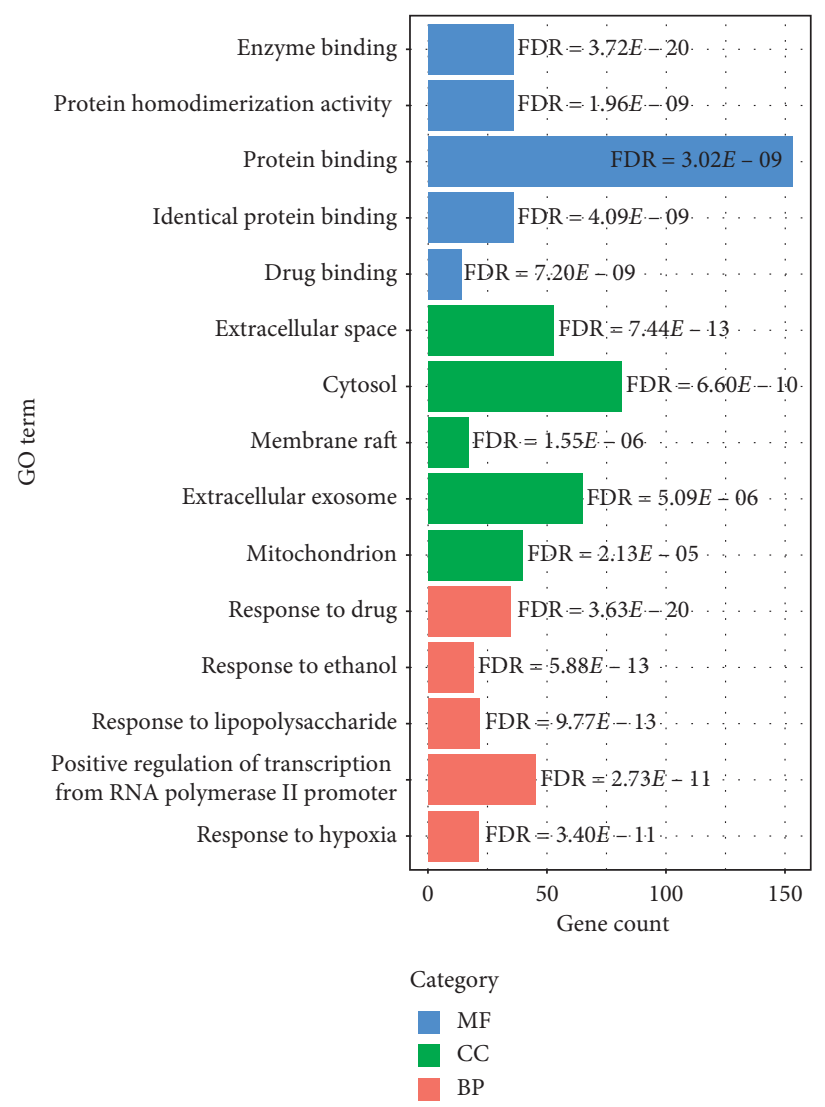

Figure 4: The barplot for GO analysis of common target genes. The top five significantly enriched GO terms in each category ranked according to FDR. FDR: false discovery rate.

TABLE 5: Results of KEGG pathway enrichment (top 20).

\begin{tabular}{lcr}
\hline Name of pathways & Gene numbers & FDR \\
\hline Hsa05200: pathways in cancer & 50 & 32 \\
Hsa05161: hepatitis B & 30 & $2.62 E-18$ \\
Hsa04151: PI3K-Akt signaling pathway & 25 & $1.86 E-05$ \\
Hsa05152: tuberculosis & 24 & $2.35 E-08$ \\
Hsa05215: prostate cancer & 24 & $2.09 E-14$ \\
Hsa04668: TNF signaling pathway & 24 & $2.42 E-12$ \\
Hsa04010: MAPK signaling pathway & 24 & $1.66 E-04$ \\
Hsa05166: HTLV-I infection & 23 & $1.78 E-04$ \\
Hsa05164: influenza A & 23 & $6.95 E-07$ \\
Hsa05205: proteoglycans in cancer & 23 & $1.00 E-05$ \\
Hsa05206: micro-RNAs in cancer & 22 & 0.005433 \\
Hsa05145: toxoplasmosis & 21 & $5.30 E-10$ \\
Hsa05160: hepatitis C & 21 & $1.92 E-07$ \\
Hsa04510: focal adhesion & 20 & $4.05 E-04$ \\
Hsa05212: pancreatic cancer & 20 & $1.71 E-12$ \\
Hsa04066: HIF-1 signaling pathway & 20 \\
Hsa05142: Chagas disease (American trypanosomiasis) & 20 & $3.78 E-09$ \\
Hsa04620: toll-like receptor signaling pathway & 20 & $1.69 E-08$ \\
Hsa04024: cAMP signaling pathway & 19 & $2.40 E-08$ \\
Hsa04014: Ras signaling pathway & 19 & 0.004254 \\
\hline
\end{tabular}

expression of CASP3 [49]. It is reported that PLC patients with high phosphorylated MAPK3 (also called ERK1) had significantly higher cancer recurrence and worse OS [50], and the impact of MAPK3 on survival was also verified by the survival curve in this study. Angiogenesis is considered as a key process in the development of cancers. VEGFA is an angiogenesis inducer in PLC, and expression of VEGFA in PLC was significantly higher than that in adjacent normal 


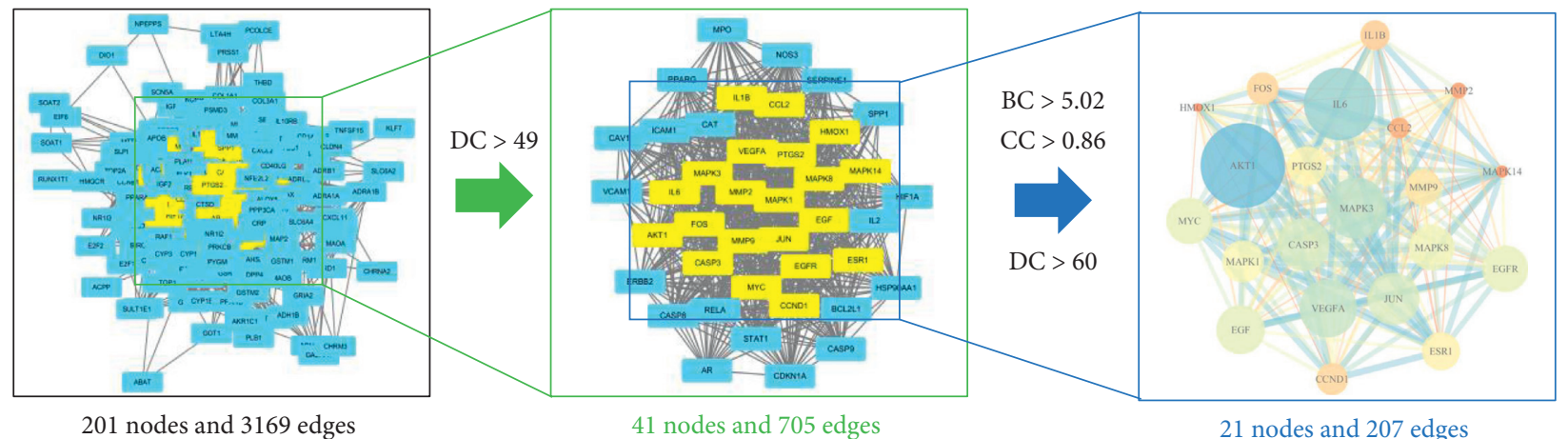

(a)

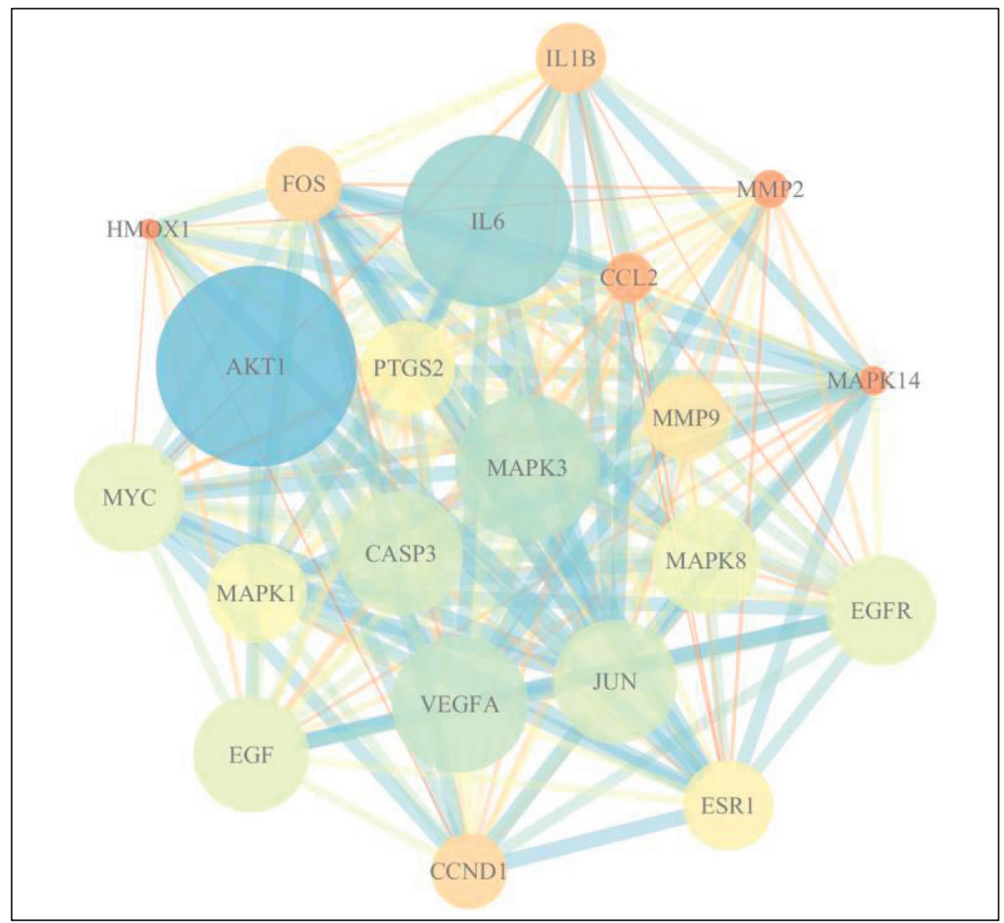

(b)

Figure 5: PPI networks of CCMMs and PLC. (a) The screening process of the target genes. The screening criteria by which the key target genes were identified were DC $>49, \mathrm{BC}>5.02$, and CC $>0.86$. (b) PPI network of CCMMs and PLC with 21 nodes and 207 edges. Nodes indicate key target genes. The size of the nodes and edges corresponds to the value of degree and combine score, respectively. The color of the nodes represents the value of degree. The darker (blue) the color, the higher the degree. DC: degree centrality; BC: betweenness centrality; CC: closeness centrality.

tissues [51]. The survival analysis demonstrated that PLC patients with high expression of VEGFA had a poor prognosis, suggesting its important role in PLC therapy. EGF, being a growth factor, plays a critical role in cell proliferation, survival, and migration by binding to its receptor EGFR. Evidence showed that high expression of EGF can induce highly malignant PLC, and activated EGF/EGFR signaling is closely related to intrahepatic metastasis [52]. Previous studies suggested that EGFR is overexpressed or mutated in PLC cells and may be closely related to the formation, invasive growth, and clinical characteristics of PLC [53, 54]. However, our data showed that high expression of EGFR is correlated with better prognosis, and further research is required to study the mechanism of this association. Last but not least, JUN and MYC are also involved in the initiation and progression of $\mathrm{HBV}$-associated PLC $[55,56]$. In general, these genes are involved in the inflammatory response, tumor neovascularization, and the occurrence and development of PLC, and some of these genes are associated with the prognosis of PLC.

In our study, the CCMMs for the treatment of PLC were identified from a large number of clinical prescriptions using the data mining approach, which may provide a useful reference for the clinical practice of TCM. Furthermore, the network pharmacology method was applied to acquire potential targets and signaling pathways of CCMMs in the treatment of PLC. According to the results of network pharmacology, CCMMs may exert 


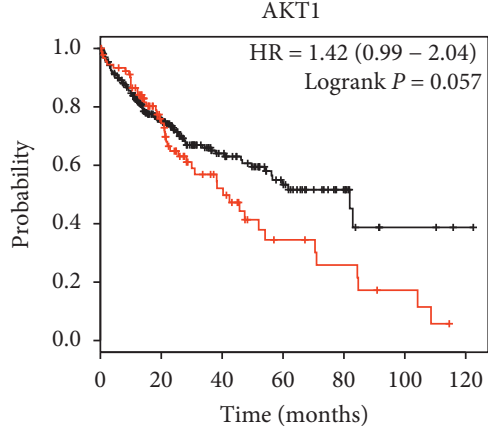

Number at risk

$\begin{array}{llllllll}\text { Low } & 272 & 132 & 62 & 33 & 13 & 3 & 1\end{array}$

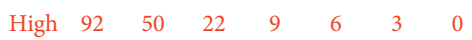

$$
\begin{array}{r}
\text { Expression } \\
- \text { Low }
\end{array}
$$

(a)

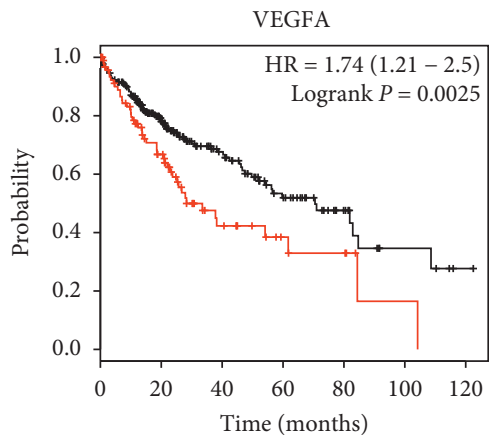

Number at risk

$\begin{array}{llllllll}\text { Low } & 268 & 134 & 68 & 35 & 14 & 5 & 1\end{array}$

$\begin{array}{llllllll}\text { High } & 96 & 48 & 16 & 7 & 5 & 1 & 0\end{array}$

$$
\begin{array}{r}
\text { Expression } \\
- \text { Low } \\
- \text { High }
\end{array}
$$

(d)

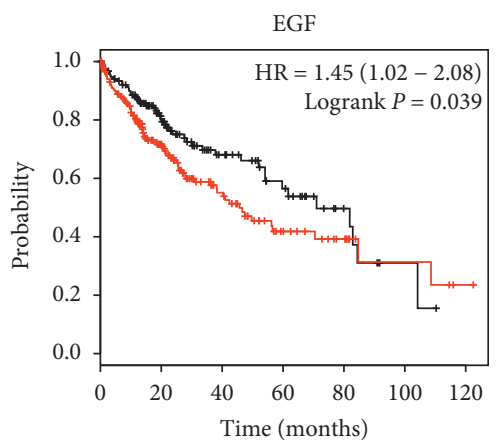

Number at risk

$\begin{array}{llllllll}\text { Low } & 157 & 84 & 40 & 22 & 8 & 2 & 0\end{array}$

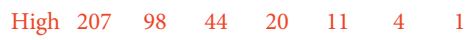

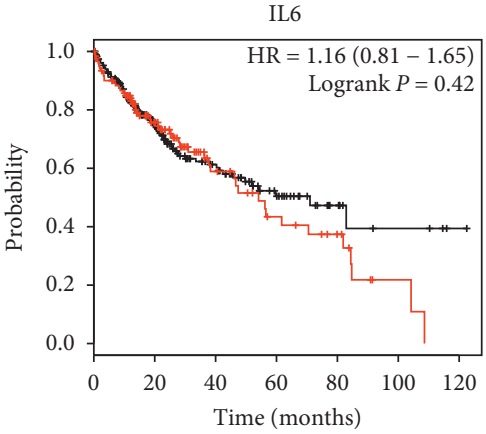

Number at risk

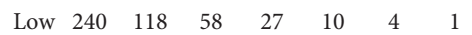

$\begin{array}{llllllll}\text { High } & 124 & 64 & 26 & 15 & 9 & 2 & 0\end{array}$

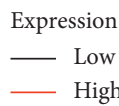

(b)

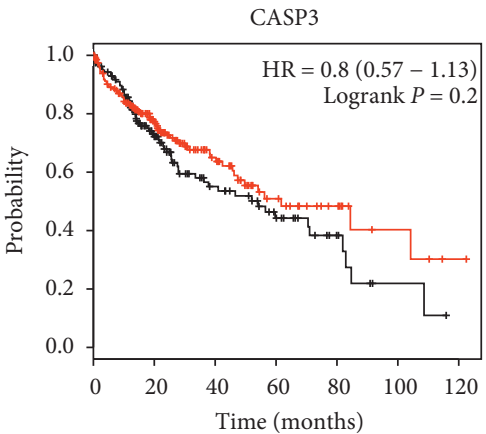

Number at risk

$\begin{array}{llllllll}\text { Low } & 163 & 75 & 36 & 21 & 8 & 2 & 0\end{array}$

$\begin{array}{llllllll}\text { High } & 201 & 107 & 48 & 21 & 11 & 4 & 1\end{array}$

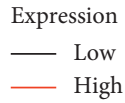

(e)

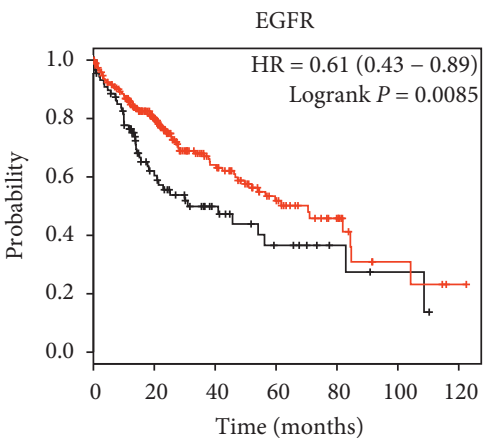

Number at risk

$\begin{array}{llllllll}\text { Low } & 91 & 39 & 19 & 9 & 4 & 2 & 0\end{array}$

$\begin{array}{llllllll}\text { High } & 273 & 143 & 65 & 33 & 15 & 4 & 1\end{array}$

$$
\begin{aligned}
\text { Expression } & \text { Expression } \\
\text { Low } & - \text { Low } \\
- \text { High } & - \text { High }
\end{aligned}
$$

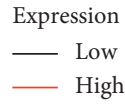

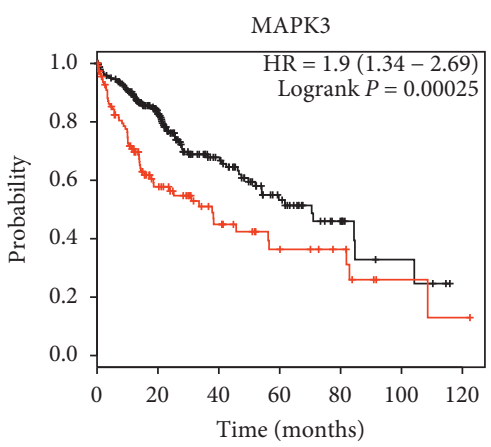

Number at risk

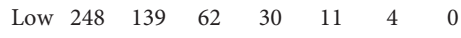

$\begin{array}{llllllll}\text { High } & 116 & 43 & 22 & 12 & 8 & 2 & 1\end{array}$

Expression

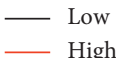

(c)

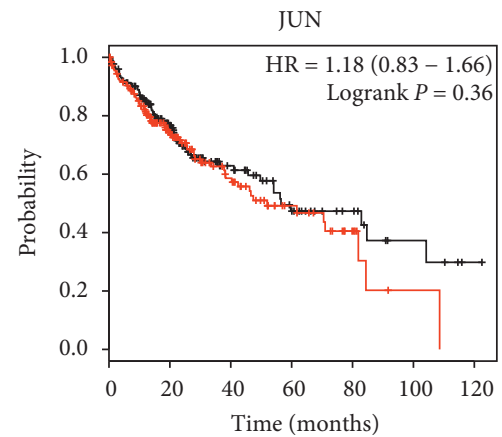

Number at risk

$\begin{array}{llllllll}\text { Low } & 181 & 97 & 41 & 22 & 12 & 5 & 1\end{array}$

$\begin{array}{llllllll}\text { High } & 183 & 85 & 43 & 20 & 7 & 1 & 0\end{array}$

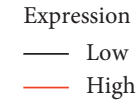

(f)

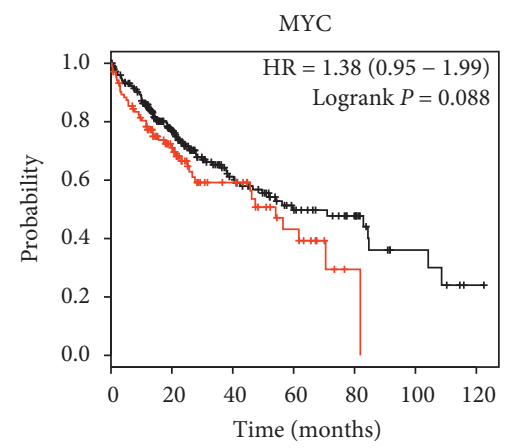

Number at risk

$\begin{array}{cccccccc}\text { Low } & 260 & 131 & 58 & 31 & 18 & 6 & 1 \\ \text { High } & 104 & 51 & 26 & 11 & 1 & 0 & 0\end{array}$

(g)

(h)

(i)

Figure 6: The prognostic values of expression levels of key genes in PLC patients. (a-i) The K-M survival curves of nine top key genes. High expression levels of MAPK3 (c), VEGFA (d), EGF (g) were associated with worse OS in PLC patients, and high expression level of EGFR (h) was correlated with longer OS. ( $\mathrm{a}, \mathrm{b}, \mathrm{e}, \mathrm{f}, \mathrm{i})$ Other genes showed no significant difference. OS: overall survival; K-M: Kaplan-Meier. 
antitumor effects on PLC by inhibiting the proliferation, invasion, and metastasis of PLC cells and by inducing cell apoptosis in PLC. Moreover, some of the pathways (e.g., PI3K-Akt, Ras/MAPK, HIF-1, TNF, and toll-like receptor pathway) and target genes (e.g., AKT1, IL6, MAPK3, EGF, and EGFR) may provide a novel approach for studying the mechanism of Chinese materia medicas in treating PLC. In the present study, the $\mathrm{K}-\mathrm{M}$ survival analysis was used to verify the prognostic value of key genes in PPI, and four key genes of CCMMs were found to be significantly related to OS in patients with PLC, which made the results of network pharmacology more reliable. However, the results in this paper depend on the reported ingredients and targets of CCMMs. With the development of experimental technology, more and more molecular targets for PLC therapy as well as new ingredients of Chinese materia medicas will be discovered, which may be helpful to enrich the results of this paper. Besides, future experiments are required to validate the findings of the present study.

\section{Conclusions}

In conclusion, based on the combination of data mining and network pharmacology, we predicted that the therapeutic effect of CCMMs on PLC may be realized by acting on multitargets and multipathways related to the occurrence and development of PLC, which laid a foundation for further experimental study.

\section{Data Availability}

The data that support the findings of this study are openly available in http://tcmspw.com/tcmsp.php, http://www. tcmip.cn/, http://bionet.ncpsb.org/batman-tcm/, https:// www.uniprot.org/, https://www.genecards.org/, https:// omim.org/, http://bioinformatics.psb.ugent.be/webtools/ Venn/, https://string-db.org/, https://david.ncifcrf.gov/, and http://kmplot.com/analysis/.

\section{Conflicts of Interest}

The authors declare no conflicts of interest.

\section{Acknowledgments}

This study was supported by Science and Technology Planning Project of Guangdong Province of China (2017A020213017).

\section{References}

[1] C. Coon, N. Berger, D. Eastwood et al., "Primary liver cancer: an NCDB analysis of overall survival and margins after hepatectomy," Annals of Surgical Oncology, vol. 27, no. 4, pp. 1156-1163, 2019.

[2] L. A. Torre, F. Bray, R. L. Siegel et al., "Global cancer statistics," CA: A Cancer Journal for Clinicians, vol. 65, no. 2, pp. 87-108, 2012.

[3] P. Luo, P. Yin, R. Hua et al., "A large-scale, multicenter serum metabolite biomarker identification study for the early detection of hepatocellular carcinoma," Hepatology, vol. 67, no. 2, pp. 662-675, 2018.

[4] F. Bray, J. Ferlay, I. Soerjomataram, R. L. Siegel, L. A. Torre, and A. Jemal, "Global cancer statistics 2018: GLOBOCAN estimates of incidence and mortality worldwide for 36 cancers in 185 countries," CA: A Cancer Journal for Clinicians, vol. 68, no. 6, pp. 394-424, 2018.

[5] H. Cheng, G. Sun, H. Chen et al., "Trends in the treatment of advanced hepatocellular carcinoma: immune checkpoint blockade immunotherapy and related combination therapies," American Journal of Cancer Research, vol. 9, no. 8, pp. 1536-1545, 2019.

[6] E. L. Yoon, J. E. Yeon, H. J. Lee et al., "Systemic cytotoxic chemotherapy of patients with advanced hepatocellular carcinoma in the era of sorafenib nonavailability," Journal of Clinical Gastroenterology, vol. 48, no. 3, pp. e22-e29, 2014.

[7] Z. Shi, T. Song, Y. Wan et al., "A systematic review and metaanalysis of traditional insect Chinese medicines combined chemotherapy for non-surgical hepatocellular carcinoma therapy," Scientific Reports, vol. 7, no. 1, p. 4355, 2017.

[8] L. Sun, P. Fahey, X. Zhu et al., "A cohort study to examine the use of Chinese herbal medicine in combination with conventional therapies for patients with hepatocellular carcinoma in China," Integrative Cancer Therapies, vol. 17, no. 3, pp. 902-911, 2018.

[9] S.-T. Kao, S.-L. Yang, C.-C. Hsieh, M.-D. Yang, T.-F. Wang, and J.-G. Lin, "Immunomodulation of bu-zhong-yi-qi-tang onIn VitroGranulocyte colony-stimulating-factor and tumor necrosis factor- $\alpha$ production by peripheral blood mononuclear cells," Immunopharmacology and Immunotoxicology, vol. 22, no. 4, pp. 711-720, 2000.

[10] Y. Hu, S. Wang, X. Wu et al., "Chinese herbal medicinederived compounds for cancer therapy: a focus on hepatocellular carcinoma," Journal of Ethnopharmacology, vol. 149, no. 3, pp. 601-612, 2013.

[11] Z. Wang, J. Li, Y. Ji et al., "Traditional herbal medicine: a review of potential of inhibitory hepatocellular carcinoma in basic research and clinical trial," Evidence-Based Complementary and Alternative Medicine, vol. 2013, Article ID 268963, 7 pages, 2013.

[12] C. Jianxin, X. Qingxia, W. Junhui, and Z. Qinhong, "A case of recurrent hepatocellular carcinoma acquiring complete remission of target lesion with treatment with traditional Chinese medicine," Integrative Cancer Therapies, vol. 16, no. 4, pp. 597-604, 2017.

[13] L. Zeng and K. Yang, "Exploring the pharmacological mechanism of Yanghe Decoction on HER2-positive breast cancer by a network pharmacology approach," Journal of Ethnopharmacology, vol. 199, pp. 68-85, 2017.

[14] A. L. Hopkins, "Network pharmacology: the next paradigm in drug discovery," Nature Chemical Biology, vol. 4, no. 11, pp. 682-690, 2008.

[15] S. H. Tang, D. Shen, and H. J. Yang, "Analysis on composition rules of Chinese patent drugs treating pain-related diseases based on data mining method," Chinese Journal of Integrative Medicine, 2017.

[16] D. Wu, X. Zhang, L. Liu, and Y. Guo, "Key CMM combinations in prescriptions for treating mastitis and working mechanism analysis based on network pharmacology," Evidence-Based Complementary and Alternative Medicine, vol. 2019, Article ID 8245071, 11 pages, 2019.

[17] J. L. Ru, P. Li, J. N. Wang et al., "TCMSP: a database of systems pharmacology for drug discovery from herbal medicines," Journal of Cheminformatics, vol. 6, 2014. 
[18] H.-Y. Xu, Y.-Q. Zhang, Z.-M. Liu et al., "ETCM: an encyclopaedia of traditional Chinese medicine," Nucleic Acids Research, vol. 47, no. D1, pp. D976-D982, 2019.

[19] Z. Liu, F. Guo, Y. Wang et al., "BATMAN-TCM: a Bioinformatics analysis tool for molecular mechANism of traditional Chinese medicine," Scientific Reports, vol. 6, p. 21146, 2016.

[20] W. Song, S. Ni, Y. Fu, and Y. Wang, "Uncovering the mechanism of Maxing Ganshi Decoction on asthma from a systematic perspective: a network pharmacology study," Scientific Reports, vol. 8, no. 1, p. 17362, 2018.

[21] G. Stelzer, N. Rosen, I. Plaschkes et al., "The GeneCards suite: from gene data mining to disease genome sequence analyses," Current Protocols in Bioinformatics, vol. 54, pp. 1.30.1-1.30.33, 2016.

[22] J. S. Amberger, C. A. Bocchini, A. F. Scott, and A. Hamosh, "OMIM.org: leveraging knowledge across phenotype-gene relationships," Nucleic Acids Research, vol. 47, no. D1, pp. D1038-D1043, 2019.

[23] G. Dennis, B. T. Sherman, D. A. Hosack et al., "DAVID: database for annotation, visualization, and integrated discovery," Genome Biology, vol. 4, no. 9, 2003.

[24] D. Szklarczyk, A. Franceschini, S. Wyder et al., "STRING v10: protein-protein interaction networks, integrated over the tree of life," Nucleic Acids Research, vol. 43, no. D1, pp. D447D452, 2015.

[25] Y. Tang, M. Li, J. Wang, Y. Pan, and F.-X. Wu, "CytoNCA: a cytoscape plugin for centrality analysis and evaluation of protein interaction networks," Biosystems, vol. 127, pp. 67-72, 2015.

[26] O. Menyhárt, Á. Nagy, and B. Győrffy, "Determining consistent prognostic biomarkers of overall survival and vascular invasion in hepatocellular carcinoma," Royal Society Open Science, vol. 5, no. 12, Article ID 181006, 2018.

[27] T. Xu, C. Ma, S. Fan et al., "Systematic understanding of the mechanism of baicalin against ischemic stroke through a network pharmacology approach," Evidence-Based Complementary and Alternative Medicine, vol. 2018, Article ID 2582843, 11 pages, 2018.

[28] S. Jia, Z. Donggeng, W. Mingyan et al., "Influence of quercetin on PI3K/AKT signal pathway of SMMC-7721 hepatic cancer cells," Chinese Journal of Experimental Traditional Medical Formulae, vol. 18, no. 18, pp. 223-226, 2012.

[29] G. Zhu, X. Liu, H. Li et al., "Kaempferol inhibits proliferation, migration, and invasion of liver cancer HepG2 cells by downregulation of microRNA-21," International Journal of Immunopathology and Pharmacology, vol. 32, 2018.

[30] E. Hernández-Aquino and P. Muriel, "Beneficial effects of naringenin in liver diseases: molecular mechanisms," World Journal of Gastroenterology, vol. 24, no. 16, pp. 1679-1707, 2018.

[31] Y. H. Choi, "Isorhamnetin induces ROS-dependent cycle arrest at G2/M phase and apoptosis in human hepatocarcinoma Hep3B cells," General physiology and biophysics, vol. 38, no. 6, pp. 473-484, 2019.

[32] Z. Zhang, Y. Xing, G. Hu, and S. Xie, "Antiproliferative effects mechanism of $\beta$-sitosterul in hepatoma HepG2 cells," China Journal of Chinese Materia Medica, vol. 36, no. 15, pp. 2145-2148, 2011.

[33] Y.-S. Kim, X.-F. Li, K.-H. Kang, B. Ryu, and S. K. Kim, "Stigmasterol isolated from marine microalgae Navicula incerta induces apoptosis in human hepatoma HepG2 cells," BMB Reports, vol. 47, no. 8, pp. 433-438, 2014.
[34] L.-X. Liu, Z. H. Liu, H. C. Jiang et al., "Gene expression profiles of hepatoma cell line HLE," World Journal of Gastroenterology, vol. 9, no. 4, pp. 683-687, 2003.

[35] R. Wu, X. Li, F. Cai et al., "Effective mechanism of Bushen Jianpi Decoction in the treatment of liver cancer based on network pharmacology," China Journal of Traditional Chinese Medicine and Pharmacy, vol. 33, no. 9, pp. 4134-4139, 2018.

[36] L. H. Zhao, X. Liu, H. X. Yan et al., "Genomic and oncogenic preference of HBV integration in hepatocellular carcinoma," Nature Communications, vol. 7, p. 12992, 2016.

[37] H.-I. Yang, S.-N. Lu, Y.-F. Liaw et al., "Hepatitis B e antigen and the risk of hepatocellular carcinoma," New England Journal of Medicine, vol. 347, no. 3, pp. 168-174, 2002.

[38] R. Chauhan, N. D. Churchill, P. M. Mulrooney-Cousins, and T. I. Michalak, "Initial sites of hepadnavirus integration into host genome in human hepatocytes and in the woodchuck model of hepatitis B-associated hepatocellular carcinoma," Oncogenesis, vol. 6, no. 4, p. e317, 2017.

[39] H. Ataseven, I. H. Bahcecioglu, N. Kuzu et al., "The levels of ghrelin, leptin, TNF- $\alpha$, and IL- 6 in liver cirrhosis and hepatocellular carcinoma due to HBV and HDV infection," Mediators of Inflammation, vol. 2006, Article ID 78380, 6 pages, 2006.

[40] F. Deng, D. Chen, X. Wei et al., "Development and validation of a prognostic classifier based on HIF-1 signaling for hepatocellular carcinoma," Aging, vol. 12, no. 4, pp. 3431-3450, 2020.

[41] X. Zhang, H. Zhang, and L. Ye, "Effects of hepatitis B virus X protein on the development of liver cancer," Journal of Laboratory and Clinical Medicine, vol. 147, no. 2, pp. 58-66, 2006.

[42] H. Nakamura, H. Aoki, O. Hino, and M. Moriyama, "HCV core protein promotes heparin binding EGF-like growth factor expression and activates Akt," Hepatology Research, vol. 41, no. 5, pp. 455-462, 2011.

[43] B. Delire and P. Stärkel, "The Ras/MAPK pathway and hepatocarcinoma: pathogenesis and therapeutic implications," European Journal of Clinical Investigation, vol. 45, no. 6, pp. 609-623, 2015.

[44] M. Benkheil, J. Paeshuyse, J. Neyts, M. Van Haele, T. Roskams, and S. Liekens, "HCV-induced EGFR-ERK signaling promotes a pro-inflammatory and pro-angiogenic signature contributing to liver cancer pathogenesis," Biochemical Pharmacology, vol. 155, pp. 305-315, 2018.

[45] X. Zhang, Z.-h. Ye, H.-w. Liang et al., "Down-regulation of miR-146a-5p and its potential targets in hepatocellular carcinoma validated by a TCGA- and GEO-based study," FEBS Open Bio, vol. 7, no. 4, pp. 504-521, 2017.

[46] L. Chen, Q.-H. Kang, Y. Chen et al., "Distinct roles of Akt1 in regulating proliferation, migration and invasion in HepG2 and HCT 116 cells," Oncology Reports, vol. 31, no. 2, pp. 737-744, 2014.

[47] G. He and M. Karin, "NF- $\kappa$ B and STAT3-key players in liver inflammation and cancer," Cell Research, vol. 21, no. 1, pp. 159-168, 2011.

[48] N. Shang, T. Bank, X. Ding et al., "Caspase-3 suppresses diethylnitrosamine-induced hepatocyte death, compensatory proliferation and hepatocarcinogenesis through inhibiting p38 activation," Cell Death \& Disease, vol. 9, no. 5, p. 558, 2018.

[49] C. Gan, Z. Zhen, B. Liang, Y. Tang, and W. Zhang, "Mechanism of total flavonoids of Ampelopsis grossedentata in inhibiting liver cancer by PI3K/Akt/p53 pathwa," Chinese 
Journal of Experimental Traditional Medical Formulae, vol. 25, no. 12, pp. 90-96, 2019.

[50] X. Gao, W. Shan, X. Liu, J. Zhang, J. Zheng, and H. Yao, "JNK1/2 and ERK1/2 provides vital clues about tumor recurrence and survival in hepatocellular carcinoma patients," Future Oncology, vol. 14, no. 24, pp. 2471-2481, 2018.

[51] J. J. Yan, Y. N. Zhang, J. Z. Liao et al., "MiR-497 suppresses angiogenesis and metastasis of hepatocellular carcinoma by inhibiting VEGFA and AEG-1," Oncotarget, vol. 6, no. 30, pp. 29527-29542, 2015.

[52] Z. Liu, D. Chen, F. Ning, J. Du, and H. Wang, "EGF is highly expressed in hepatocellular carcinoma (HCC) and promotes motility of HCC cells via fibronectin," Journal of Cellular Biochemistry, vol. 119, no. 5, pp. 4170-4183, 2018.

[53] R. Panvichian, A. Tantiwetrueangdet, P. Sornmayura, and S. Leelaudomlipi, "Missense mutations in exons 1824 of EGFR in hepatocellular carcinoma tissues," BioMed Research International, vol. 2015, Article ID 171845, 7 pages, 2015.

[54] K. Komposch and M. Sibilia, "EGFR signaling in liver diseases," International Journal of Molecular Sciences, vol. 17, no. 1, 2015.

[55] L. Guo, Y. Guo, S. Xiao, and X. Shi, "Protein kinase p-JNK is correlated with the activation of AP-1 and its associated Jun family proteins in hepatocellular carcinoma," Life Sciences, vol. 77, no. 15, pp. 1869-1878, 2005.

[56] H. Tsuchiya, M. Amisaki, A. Takenaga et al., "HBx and c-MYC cooperate to induce URI1 expression in HBV-related hepatocellular carcinoma," International Journal of Molecular Sciences, vol. 20, no. 22, 2019. 